\title{
Chapter 12 \\ Knowledge-Based UML Dynamic Models \\ Generation from Enterprise Model \\ in Hospital Information Management \\ Process Example
}

\section{Ilona Veitaite and Audrius Lopata}

\begin{abstract}
The main purpose of this paper is to present knowledge-based Enterprise model (EM) sufficiency as data repository for Unified Modelling Language (UML) models generation. UML models are one of the most usable modelling languages in system lifecycle design stage, despite the problem domain of the system. UML models can be generated from Enterprise Model by using particular transformation algorithms presented in previous researches. Generation process from Enterprise model is represented by certain Hospital Information Management process example. Generated UML dynamic Use Case, Activity, Sequence and State models of different perspectives of Hospital Information Management process prove sufficiency of stored information in Enterprise model.
\end{abstract}

Keywords UML • Enterprise model • Transformation algorithm • Knowledge-based IS engineering $\cdot$ Hospital IS management

\subsection{Introduction}

Despite the progress of all information technologies, information system (IS) engineering process still challenges professionals of this field: analysts, designers, researchers and etc. Enterprise modelling makes giant impact to successful information system design process. There are many Enterprise models and Enterprise modelling methodologies, which are applied in different ways and various types of models are built based on chosen Enterprise model [1,2].

\footnotetext{
I. Veitaite $(\varangle)$

Institute of Social Sciences and Applied Informatics, Vilnius University, Muitinès g. 8, 44280

Kaunas, Lithuania

e-mail: ilona.veitaite@knf.vu.lt
}

\author{
A. Lopata \\ Kaunas University of Technology, Studentụ g. 50, 51368 Kaunas, Lithuania \\ e-mail: audrius.lopata@ktu.lt


Unified Modelling Language is a highly accepted among IS analysts and designers and is commonly used for IS design. It is used as standard notation to represent designed information system from different views, it provides information in both: structural and behavioural perspectives. Correctly created UML models of any problem domain can be the background for code generation and ensure the success of final IS version [1, 3-5].

Enterprise model can be used as the background for UML models. Correct UML models can be created only then, when gathered into Enterprise Model data is verified, validated and have enough quality. Data gathering process should be done under analysts and experts supervision. Enterprise model with verified and validated data of particular problem domain fully serves all necessary data. Using this data UML models can be generated from Enterprise model through transformation algorithms and after generation process these models are main source for further IS development life cycle stage [3, 4, 6-9].

\subsection{Structure of Knowledge-Based Enterprise Model}

EMM is formally defined EM structure, which consists of a formalized EM in line with the general principles of control theory. EM is the main source of the necessary knowledge of the particular business domain for IS engineering and IS re-engineering processes (Fig. 12.1) [6, 10].

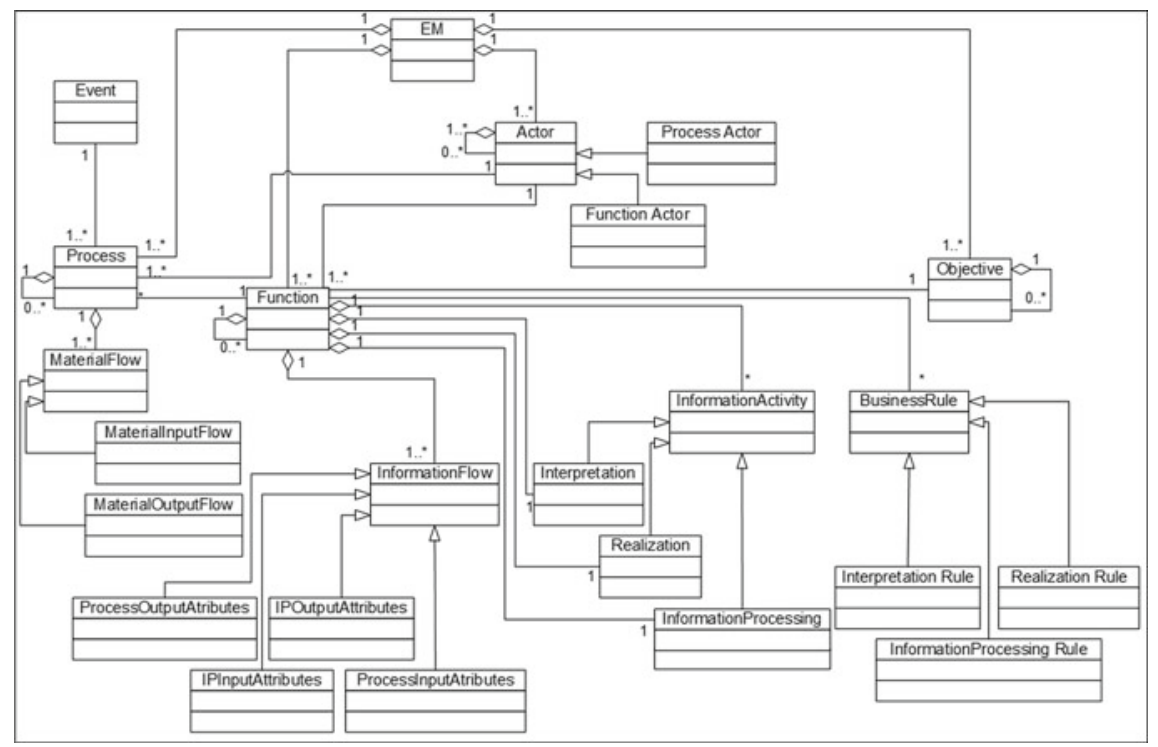

Fig. 12.1 Enterprise meta-model class diagram $[6,9,10]$ 
EM class model has twenty-three classes. Essential classes are Process, Function and Actor. Class Process, Function, Actor and Objective can have an internal hierarchical structure. These relationships is presented as aggregation relationship. Class Process is linked with the class MaterialFlow as aggregation relationship. Class MaterialFlow is linked with the classes MaterialInputFlow and MaterialOutputFlow as generalization relationship. Class Process is linked with Classes Function, Actor and Event as association relationship. Class Function is linked with classes InformationFlow, InformationActivity, Interpretation, InformationProcessing and Realization as aggregation relationship. These relationships define the internal composition of the Class Function. Class InformationFlow is linked with ProcessOutputAtributes, ProcessInputAtributes, IPInputAttributes and IPOutputAttributs as generalization relationship. Class InformationActivity is linked with Interpretation, InformationProcessing and Realization as generalization relationship. Class Function linked with classes Actor, Objective and BusinessRule as association relationship. Class BusinessRule is linked with Interpretation Rule, Realization Rule, InformationProcessing Rule as generalization relationship. Class Actor is linked with Function Actor and Process Actor as generalization relationship [3, 6, 10, 11].

\subsection{Transformation Algorithms of UML Models from Enterprise Model}

Each of structural or behavioural UML models can be generated through transformation algorithm and each of models has separate transformation algorithm. These transformation algorithms are presented in previous researches. Main focus of researches is dedicated for generation behavioural or dynamic UML models, because they are more complex and variable [9,11-13]. To have better understanding of transformation algorithm itself, top level transformation algorithm of UML models generation from EM process is presented in the figure (Fig. 12.2) and described step by step [9, 11-13].

- Step 1: Particular UML model for generation from EM process is identified and selected.

- Step 2: If the particular UML model for generation from EM process is selected then algorithm process is continued, else the particular UML model for generation from EM process must be selected.

- Step 3: First element from EM is selected for UML model, identified previously, generation process.

- Step 4: If the selected EM element is initial UML model element, then initial element is generated, else the other EM element must be selected (the selected element must be initial element).

- Step 5: The element related to the initial element is selected from Enterprise model. 


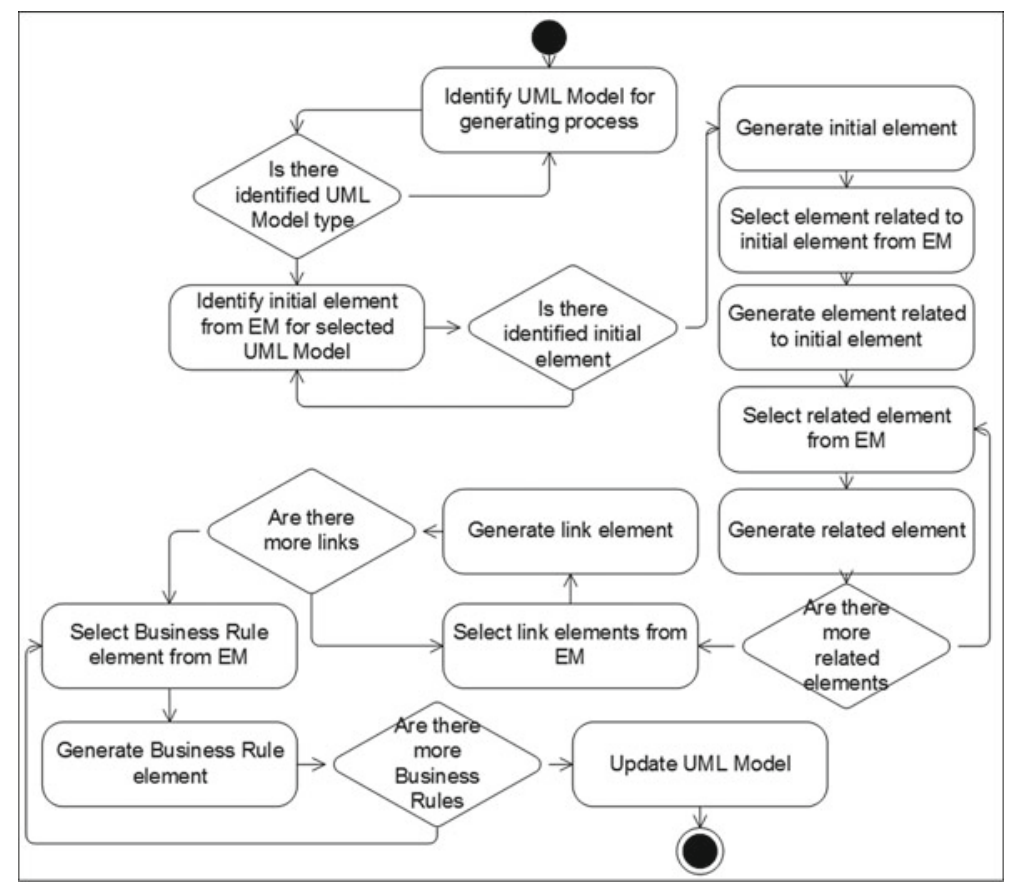

Fig. 12.2 The top level transformation algorithm of UML models generation from EM process [9, $11-13]$

- Step 6: The element related to the initial element is generated as UML model element.

- Step 7: The element related to the previous element is selected from Enterprise model.

- Step 8: The element related to the previous element is generated as UML model element.

- Step 9: If there are more related elements, then they are selected from EM and generated as UML model elements one by one, else the link element is selected from Enterprise model.

- Step 10: The link element is generated as UML model element.

- Step 11: If there are more links, then they are selected from EM and generated as UML model elements one by one, else the Business Rule element is selected from Enterprise model.

- Step 12: The Business Rule element is generated as UML model element.

- Step 13: If there are more Business Rules, then they are selected from EM and generated as UML model elements one by one, else the generated UML model is updated with all elements, links and constraints.

- Step 14: Generation process is finished. 
Table 12.1 Description of knowledge stored in Enterprise model

\begin{tabular}{l|l}
\hline Enterprise model element & Description \\
\hline Actor & $\begin{array}{l}\text { In actor element can be stored information related with process or } \\
\text { function executor. Actor element is responsible of information } \\
\text { related with the process or function participant, it can be person, } \\
\text { group of persons, subject such as an IS, subsystem, module and etc }\end{array}$ \\
\hline Process, function & $\begin{array}{l}\text { In process or function elements can be stored all information } \\
\text { related with any user, entity, object, subject and its behaviour. } \\
\text { Process or function element is responsible of information related } \\
\text { with any operation, activity, status change, movement which is } \\
\text { implemented by any actor, entity, participant and etc }\end{array}$ \\
\hline Information flow & $\begin{array}{l}\text { In Information Flow element can be stored diverse information } \\
\text { flow types, such as Information input and output attributes or/and } \\
\text { process input and output attributes. Information Flow element is } \\
\text { responsible of information related with each element input and } \\
\text { output attributes, details which make impact on other elements, } \\
\text { their state or status }\end{array}$ \\
\hline Business rule & $\begin{array}{l}\text { In Business Rule element can be stored different rules such as } \\
\text { interpretation, realization or/and information processing. Business } \\
\text { rule element is responsible of information about how different } \\
\text { elements in IS design phase are related; what restrictions and } \\
\text { restraints are applied to these elements }\end{array}$ \\
\hline
\end{tabular}

Table 12.1 presents part of Enterprise model elements and their descriptions in order to describe elements, which are necessary in this particular research.

\subsection{Generated UML Models of Hospital Information Management Process Example}

The Hospital intended to manage outside patients is the object of presented example. In this institution a doctor is only associated with one specialized hospital department (cardiology, pediatrics, etc.) at a time. Each doctor has a visiting time and day in a week.

At reception the patient data is entered and the necessary fees are also taken. The patient is tracked on the basis of the ID number which is generated automatically.

Usually a patient can visit the doctors in two possible ways: directly selecting a doctor or by getting admitted to the hospital.

A doctor can prescribe tests based on the patient's described condition. The patient visits the laboratory to get done the tests prescribed by the doctor. The reports of the tests are given to the patient. The payments related to the tests are done at the reception. According the reports, the doctor prescribes the patient medicines or further tests, if they are needed or is asked to get admitted in hospital. 
If available a patient is admitted into a ward of a particular department as per the doctor's prescription. The number of available wards is limited and if there is no free ward the admission of the patient is rescheduled.

Also in case of the prescription of the doctor the patient is operated on a scheduled date and time as decided by the doctor who is responsible for the operation.

After the finishing of the treatment a patient may get discharged on an advice of his doctor and upon the full payment of all due charges at the reception. On payment of complete dues the reception generates a discharge card for the patient.

All data of particular problem domain, in this case, Hospital Information Management data is stored in Enterprise Model described previously. Stored information in Enterprise model is already verified and validated by expert and analyst, so it is ready to use for UML model generation.

\subsubsection{UML Use Case Model of Hospital Information Management Process Example}

A UML Use Case model is the initial form to identify and present system requirements for a new IS underdeveloped. Use cases identify the expected behaviour - what should be done, and not the exact method of how it should be done. Main advantage of use case modelling is that it assists to design a system from the end user's view. It is a powerful technique for communicating system behaviour in the user's conditions by specifying all externally visible system behaviour $[3,7,8]$.

Table 12.2 presents UML Use Case model elements generated from Enterprise model of Hospital Information Management process example. In Enterprise Model all information related with actors, their functions and relationships between these functions is stored. There are four actors: Patient, Doctor, Receptionist and Laboratory Assistant; Receptionist is related with five use cases; Laboratory Assistant-with one use case; Doctor is related with three uses cases and Patient is related with seven use cases. Four use cases includes some additional use cases, six relationships in total. These elements and their relationships are presented in the next figure.

Figure 12.3 presents UML Use Case model of Hospital Information Management process example generated step by step from Enterprise Model through UML Use Case transformation algorithm.

\subsubsection{UML Activity Models of Hospital Information Management Process Example}

UML Activity model describes how activities are coordinated, activities dependence from the actor or previous activity. It provides a service which can be in various levels of abstraction. Usually, an event needs to be gained by some operations, particularly 
Table 12.2 UML use case model elements generated from enterprise model of hospital information management process example $[3,7,8]$

\begin{tabular}{|c|c|c|c|}
\hline $\begin{array}{l}\text { Enterprise model } \\
\text { element }\end{array}$ & $\begin{array}{l}\text { UML use case model } \\
\text { element }\end{array}$ & $\begin{array}{l}\text { Hospital information } \\
\text { management process } \\
\text { example }\end{array}$ & Description \\
\hline \multirow[t]{4}{*}{ Actor } & \multirow[t]{4}{*}{ Actor } & Patient & \multirow{4}{*}{$\begin{array}{l}\text { There are four actors, } \\
\text { each of them is } \\
\text { behavioural classifier } \\
\text { which defines a role } \\
\text { played in particular } \\
\text { example }\end{array}$} \\
\hline & & Doctor & \\
\hline & & Receptionist & \\
\hline & & Laboratory assistant & \\
\hline \multirow[t]{14}{*}{ Process, function } & \multirow[t]{14}{*}{ Use case } & $\begin{array}{l}\text { Laboratory visit for } \\
\text { the test }\end{array}$ & \multirow{14}{*}{$\begin{array}{l}\text { There are fourteen use } \\
\text { cases, each use case is a } \\
\text { type of behavioural } \\
\text { classifier that describes a } \\
\text { unit of functionality } \\
\text { performed by three } \\
\text { actors }\end{array}$} \\
\hline & & Test report generation & \\
\hline & & $\begin{array}{l}\text { Payment for the test at } \\
\text { reception }\end{array}$ & \\
\hline & & $\begin{array}{l}\text { Registration for } \\
\text { treatment }\end{array}$ & \\
\hline & & ID generation & \\
\hline & & Fee payment & \\
\hline & & Admission to ward & \\
\hline & & Discharging & \\
\hline & & Account settlement & \\
\hline & & $\begin{array}{l}\text { Discharging card } \\
\text { generation }\end{array}$ & \\
\hline & & Test prescription & \\
\hline & & Test report analysis & \\
\hline & & $\begin{array}{l}\text { Prescription for } \\
\text { medicines }\end{array}$ & \\
\hline & & Operation performing & \\
\hline Business rule & Include & Six include elements & $\begin{array}{l}\text { There are six include } \\
\text { elements, each include is } \\
\text { a directed relationship } \\
\text { between two use cases } \\
\text { which is used to } \\
\text { demonstrate that } \\
\text { behaviour of the } \\
\text { included use case is } \\
\text { inserted into the } \\
\text { behaviour of the } \\
\text { including use case }\end{array}$ \\
\hline
\end{tabular}




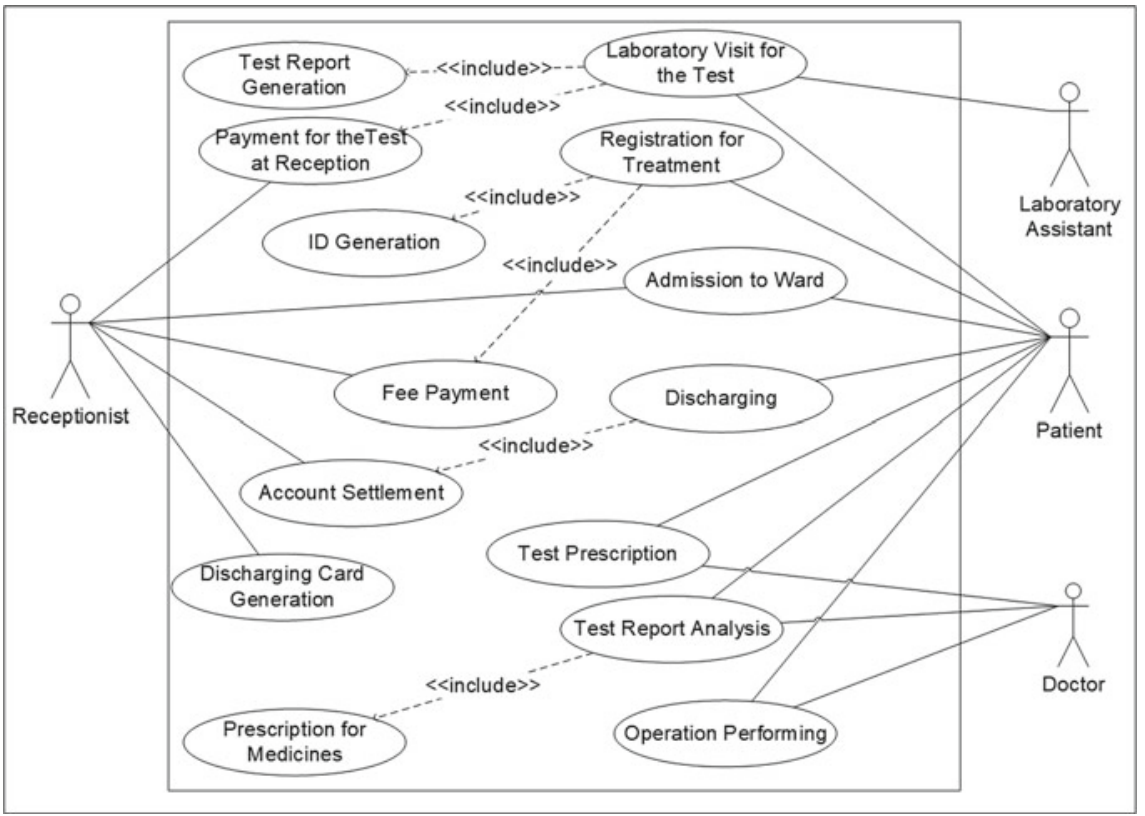

Fig. 12.3 UML use case model of hospital information management process example

where the operation is intended to gain a number of different things that require coordination, or how the events in a single use case relate to one another, especially, use cases where activities may overlap and require coordination [3, 7, 8].

According previously described UML Use Case model there is possible to identify at least five different UML Activity models: Patient Registration, Ward Assignation, Medical Tests, Treatment Process and Discharging.

\section{UML Activity Model: Patient Registration}

First UML Activity Model generated from EM is Patient Registration, where two participants - actors take part: Patient and Receptionist.

Table 12.3 presents UML Activity model elements generated from Enterprise model of Hospital Information Management process example, Registration part. Actor-first UML Activity model partition Patient starts registration process: visits reception, provides personal data, Actor-second partition Receptionist enters patient's data and provides patient's ID number, last activity Fee Payment is related with first partition, Patient pays the fee and registration process ends.

Figure 12.4 presents UML Activity Model of Hospital Information Management process example, Registration part generated step by step from Enterprise model through UML Activity model transformation algorithm [9]. 
Table 12.3 UML activity model elements generated from enterprise model of hospital information management process example, registration part $[3,7,8]$

\begin{tabular}{|c|c|c|c|}
\hline $\begin{array}{l}\text { Enterprise model } \\
\text { element }\end{array}$ & $\begin{array}{l}\text { UML activity model } \\
\text { element }\end{array}$ & $\begin{array}{l}\text { Hospital information } \\
\text { management process } \\
\text { example }\end{array}$ & Description \\
\hline \multirow[t]{2}{*}{ Actor } & \multirow[t]{2}{*}{ Partition } & Patient & \multirow{2}{*}{$\begin{array}{l}\text { There are two } \\
\text { partitions and } \\
\text { activities are related } \\
\text { with these actors }\end{array}$} \\
\hline & & Receptionist & \\
\hline \multirow[t]{5}{*}{ Function, process } & \multirow[t]{5}{*}{ Activity } & Reception visit & \multirow{5}{*}{$\begin{array}{l}\text { There are five } \\
\text { activities directly } \\
\text { related with two } \\
\text { partitions: } \\
\text { patient-three } \\
\text { activities, } \\
\text { receptionist-two. } \\
\text { They represent a } \\
\text { parameterized } \\
\text { behaviour as } \\
\text { coordinated flow of } \\
\text { actions }\end{array}$} \\
\hline & & Personal data provision & \\
\hline & & $\begin{array}{l}\text { Data entering into } \\
\text { system }\end{array}$ & \\
\hline & & Patient ID generation & \\
\hline & & fee payment & \\
\hline \multirow[t]{2}{*}{ Business rules } & \multirow[t]{2}{*}{ Control nodes } & Initial node & \multirow{2}{*}{$\begin{array}{l}\text { There are two control } \\
\text { nodes: one } \\
\text { node-initial node in } \\
\text { the beginning; final } \\
\text { node in the end of the } \\
\text { process }\end{array}$} \\
\hline & & Final node & \\
\hline
\end{tabular}

Fig. 12.4 UML activity model of hospital information management process example. registration

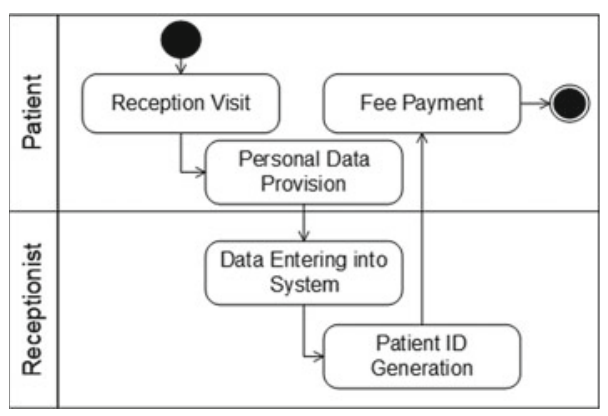

\section{UML Activity Model: Ward Assignation}

Second UML Activity Model generated from EM is Ward Assignation, where two participants-actors take part: Patient and Receptionist.

Table 12.4 presents UML Activity model elements generated from Enterprise model of Hospital Information Management process example, Ward Assignation 
Table 12.4 UML activity model elements generated from enterprise model of hospital information management process example, ward assignation part $[3,7,8]$

\begin{tabular}{|c|c|c|c|}
\hline $\begin{array}{l}\text { Enterprise model } \\
\text { element }\end{array}$ & $\begin{array}{l}\text { UML activity model } \\
\text { element }\end{array}$ & $\begin{array}{l}\text { Hospital information } \\
\text { management process } \\
\text { example }\end{array}$ & Description \\
\hline \multirow[t]{2}{*}{ Actor } & \multirow[t]{2}{*}{ Partition } & Patient & \multirow{2}{*}{$\begin{array}{l}\text { There are two partitions } \\
\text { and activities are } \\
\text { related with these actors }\end{array}$} \\
\hline & & Receptionist & \\
\hline \multirow[t]{4}{*}{ Function, process } & \multirow[t]{4}{*}{ Activity } & Ward availability check & \multirow{4}{*}{$\begin{array}{l}\text { There are four activities } \\
\text { directly related with } \\
\text { two partitions: } \\
\text { patient—one activity, } \\
\text { receptionist—-three. } \\
\text { They represent a } \\
\text { parameterized } \\
\text { behaviour as } \\
\text { coordinated flow of } \\
\text { actions }\end{array}$} \\
\hline & & Provision of new dates & \\
\hline & & Ward assignment & \\
\hline & & New dates inquiry & \\
\hline \multirow[t]{2}{*}{ Information flow } & \multirow[t]{2}{*}{ Object flow edge } & $\begin{array}{l}\text { Ward assignation } \\
\text { details to patient }\end{array}$ & \multirow{2}{*}{$\begin{array}{l}\text { There are two object } \\
\text { flow edges which are } \\
\text { activity edges used to } \\
\text { show data flow between } \\
\text { activities }\end{array}$} \\
\hline & & $\begin{array}{l}\text { Ward information } \\
\text { update to reception }\end{array}$ & \\
\hline \multirow[t]{5}{*}{ Business rules } & \multirow[t]{5}{*}{ Control nodes } & Initial node & \multirow{5}{*}{$\begin{array}{l}\text { There are five control } \\
\text { nodes: one } \\
\text { node-initial node in } \\
\text { the beginning; decision } \\
\text { node-for ward } \\
\text { availability check; join } \\
\text { and fork nodes-to } \\
\text { relate object flow edges, } \\
\text { final node in the end of } \\
\text { the process }\end{array}$} \\
\hline & & Decision node & \\
\hline & & Join node & \\
\hline & & Fork node & \\
\hline & & Final node & \\
\hline
\end{tabular}

part. Actor-first UML Activity model partition Receptionist starts Ward Assignation process: Checks ward availability, assigns it, or inquires for new dates, because there are no free wards, Actor-second partition Patient provides new date for ward assignation, last activities are related with first partition, Receptionist prepares information for patient and updates information in Reception and process ends.

Figure 12.5 presents UML Activity Model of Hospital Information Management process example, Ward Assignation part generated step by step from Enterprise model through UML Activity model transformation algorithm [9].

\section{UML Activity Model: Medical Tests}

Third UML Activity Model generated from EM is Medical Tests, where three participants—actors take part: Patient, Laboratory Assistant and Receptionist. 


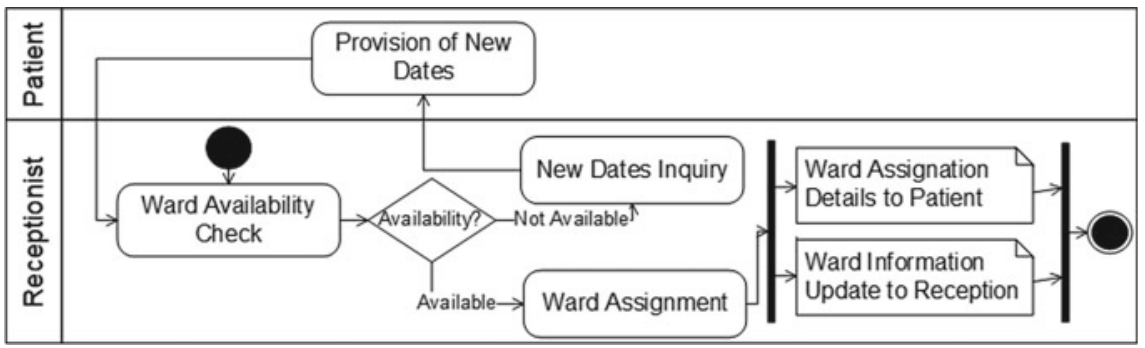

Fig. 12.5 UML activity model of hospital information management process example. Ward assignation

Table 12.5 UML activity model elements generated from enterprise model of hospital information management process example, medical tests part $[3,7,8]$

\begin{tabular}{|c|c|c|c|}
\hline $\begin{array}{l}\text { Enterprise model } \\
\text { element }\end{array}$ & $\begin{array}{l}\text { UML activity model } \\
\text { element }\end{array}$ & $\begin{array}{l}\text { Hospital information } \\
\text { management process } \\
\text { example }\end{array}$ & Description \\
\hline \multirow[t]{3}{*}{ Actor } & \multirow[t]{3}{*}{ Partition } & Patient & \multirow{3}{*}{$\begin{array}{l}\text { There are three partitions } \\
\text { and activities are related } \\
\text { with these actors }\end{array}$} \\
\hline & & Laboratory assistant & \\
\hline & & receptionist & \\
\hline \multirow[t]{10}{*}{ Function, process } & \multirow[t]{10}{*}{ Activity } & $\begin{array}{l}\text { Laboratory visit for } \\
\text { test }\end{array}$ & \multirow{10}{*}{$\begin{array}{l}\text { There are ten activities } \\
\text { directly related with } \\
\text { three partitions: } \\
\text { patient—four activities, } \\
\text { laboratory } \\
\text { assistant-five activities, } \\
\text { receptionist—one. They } \\
\text { represent a } \\
\text { parameterized behaviour } \\
\text { as coordinated flow of } \\
\text { actions }\end{array}$} \\
\hline & & $\begin{array}{l}\text { Doctor's prescription } \\
\text { check }\end{array}$ & \\
\hline & & Sample inquiry & \\
\hline & & Sample provision & \\
\hline & & Performing of the test & \\
\hline & & $\begin{array}{l}\text { Payment order } \\
\text { generation }\end{array}$ & \\
\hline & & Report generation & \\
\hline & & Fee payment & \\
\hline & & Issuing receipt & \\
\hline & & $\begin{array}{l}\text { Payment receipt } \\
\text { provision }\end{array}$ & \\
\hline \multirow[t]{4}{*}{ Business rules } & \multirow[t]{4}{*}{ Control nodes } & Initial node & \multirow{4}{*}{$\begin{array}{l}\text { There are four control } \\
\text { nodes: one node-initial } \\
\text { node in the beginning; } \\
\text { join and fork nodes-to } \\
\text { relate additional } \\
\text { activities; final node in } \\
\text { the end of the process }\end{array}$} \\
\hline & & Join node & \\
\hline & & Fork node & \\
\hline & & Final node & \\
\hline
\end{tabular}




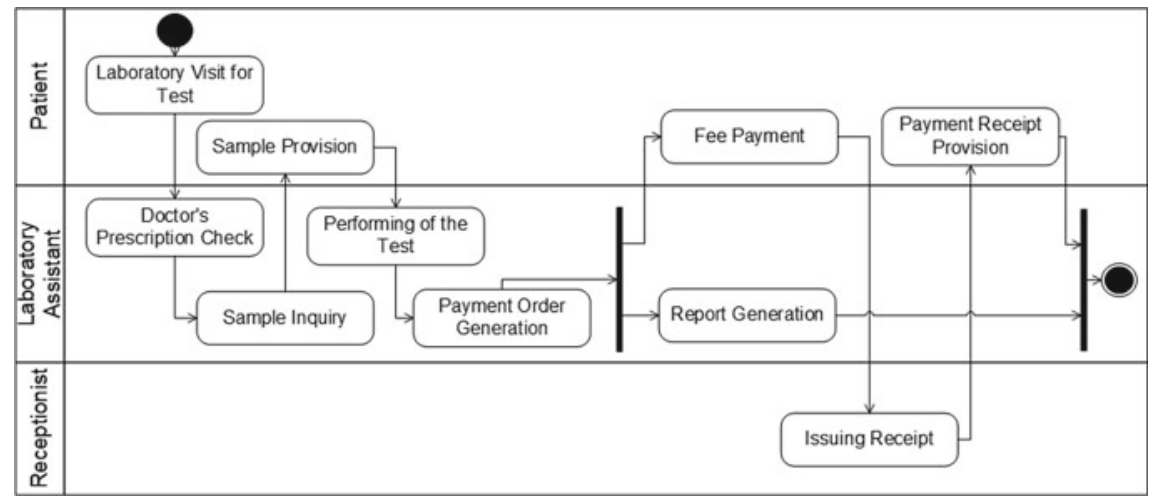

Fig. 12.6 UML activity model of hospital information management process example. Medical tests

Table 12.5 presents UML Activity model elements generated from Enterprise model of Hospital Information Management process example, Medical Tests part. Actor-first UML Activity model partition Patient starts Medical Tests process: visits laboratory and provides sample after inquiry, Actor-second partition Laboratory checks doctor's prescription, inquires for sample, performs test, generates payment order and prepares report for the doctor; Actor-third partition Receptionist confirms payment form the patient and provides receipt; Patient makes payment and after payment confirmation receives receipt and process ends.

Figure 12.6 presents UML Activity Model of Hospital Information Management process example, Medical Tests part generated step by step from Enterprise model through UML Activity model transformation algorithm [9].

\section{UML Activity Model: Treatment Process}

Fourth UML Activity Model generated from EM is Medical Tests, where two participants - actors take part: Patient and Doctor.

Table 12.6 presents UML Activity model elements generated from Enterprise model of Hospital Information Management process example, Treatment Process part. Actor-first UML Activity model partition Doctor starts Treatment Process: meets the patient, analyses provided test reports, regarding test results decides to discharge patient or continue treatment process. Doctor decides if there is need to do more tests or not, assigns treatment method medicine or operational intervention, after actor-second partition Patient confirmation, Doctor performs operation and process ends.

Figure 12.7 presents UML Activity Model of Hospital Information Management process example, Treatment Process part generated step by step from Enterprise model through UML Activity model transformation algorithm [9]. 
Table 12.6 UML activity model elements generated from enterprise model of hospital information management process example, treatment process part $[3,7,8]$

\begin{tabular}{|c|c|c|c|}
\hline $\begin{array}{l}\text { Enterprise model } \\
\text { element }\end{array}$ & $\begin{array}{l}\text { UML activity model } \\
\text { element }\end{array}$ & $\begin{array}{l}\text { Hospital information } \\
\text { management process } \\
\text { example }\end{array}$ & Description \\
\hline \multirow[t]{2}{*}{ Actor } & \multirow[t]{2}{*}{ Partition } & Patient & \multirow{2}{*}{$\begin{array}{l}\text { There are two partitions } \\
\text { and activities are related } \\
\text { with these actors }\end{array}$} \\
\hline & & Doctor & \\
\hline \multirow[t]{10}{*}{ Function, process } & \multirow[t]{10}{*}{ Activity } & Patient visit & \multirow{10}{*}{$\begin{array}{l}\text { There are ten activities } \\
\text { directly related with two } \\
\text { partitions: patient-two } \\
\text { activities, } \\
\text { doctor-eight. They } \\
\text { represent a } \\
\text { parameterized } \\
\text { behaviour as } \\
\text { coordinated flow of } \\
\text { actions }\end{array}$} \\
\hline & & Test report provision & \\
\hline & & Report analysis & \\
\hline & & Issuing discharge & \\
\hline & & $\begin{array}{l}\text { Test requirements } \\
\text { check }\end{array}$ & \\
\hline & & Test prescription & \\
\hline & & $\begin{array}{l}\text { Treatment requirement } \\
\text { check }\end{array}$ & \\
\hline & & Operation scheduling & \\
\hline & & $\begin{array}{l}\text { Confirmation of } \\
\text { operation }\end{array}$ & \\
\hline & & Performing operation & \\
\hline \multirow[t]{3}{*}{ Business rules } & \multirow[t]{3}{*}{ Control nodes } & Initial node & \multirow{3}{*}{$\begin{array}{l}\text { There are five control } \\
\text { nodes: one } \\
\text { node-initial node in } \\
\text { the beginning; three } \\
\text { decision nodes-for test } \\
\text { report status, for more } \\
\text { tests possibility, for } \\
\text { treatment type; final } \\
\text { node in the end of the } \\
\text { process }\end{array}$} \\
\hline & & Decision nodes & \\
\hline & & Final node & \\
\hline
\end{tabular}

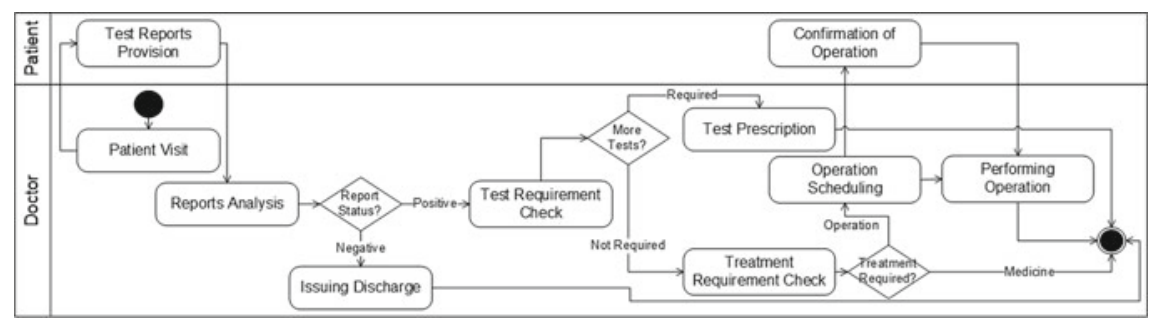

Fig. 12.7 UML activity model of hospital information management process example. Treatment process 
Table 12.7 UML activity model elements generated from enterprise model of hospital information management process example, discharging part $[3,7,8]$

\begin{tabular}{|c|c|c|c|}
\hline $\begin{array}{l}\text { Enterprise model } \\
\text { element }\end{array}$ & $\begin{array}{l}\text { UML activity model } \\
\text { element }\end{array}$ & $\begin{array}{l}\text { Hospital information } \\
\text { management process } \\
\text { example }\end{array}$ & Description \\
\hline \multirow[t]{2}{*}{ Actor } & \multirow[t]{2}{*}{ Partition } & Patient & \multirow{2}{*}{$\begin{array}{l}\text { There are two partitions } \\
\text { and activities are related } \\
\text { with these actors }\end{array}$} \\
\hline & & Receptionist & \\
\hline \multirow[t]{6}{*}{ Function, process } & \multirow[t]{6}{*}{ Activity } & $\begin{array}{l}\text { Approaching with } \\
\text { discharge advice }\end{array}$ & \multirow{6}{*}{$\begin{array}{l}\text { There are six activities } \\
\text { directly related with two } \\
\text { partitions: patient-two } \\
\text { activities, } \\
\text { receptionist-four. They } \\
\text { represent a } \\
\text { parameterized behaviour } \\
\text { as coordinated flow of } \\
\text { actions }\end{array}$} \\
\hline & & Data check & \\
\hline & & $\begin{array}{l}\text { Discharge card } \\
\text { generation }\end{array}$ & \\
\hline & & Payment check order & \\
\hline & & Due amount payment & \\
\hline & & $\begin{array}{l}\text { Discharge card } \\
\text { provision }\end{array}$ & \\
\hline \multirow[t]{3}{*}{ Business rules } & \multirow[t]{3}{*}{ Control nodes } & Initial node & \multirow{3}{*}{$\begin{array}{l}\text { There are two control } \\
\text { nodes: one node-initial } \\
\text { node in the beginning; } \\
\text { decision node-for } \\
\text { payment status final } \\
\text { node in the end of the } \\
\text { process }\end{array}$} \\
\hline & & Decision node & \\
\hline & & Final node & \\
\hline
\end{tabular}

\section{UML Activity Model: Discharging}

Fifth UML Activity Model generated from EM is Discharging, where two participants-actors take part: Patient and Receptionist.

Table 12.7 presents UML Activity model elements generated from Enterprise model of Hospital Information Management process example, Discharging part. Actor-first UML Activity model partition Patient starts discharging process: approaches with discharge advice from the doctor, Actor-second partition Receptionist checks data, generate discharge card, check payment status, after patient makes payment, provides discharge card and process ends.

Figure 12.8 presents UML Activity Model of Hospital Information Management process example, Discharging part generated step by step from Enterprise model through UML Activity model transformation algorithm [9]. 


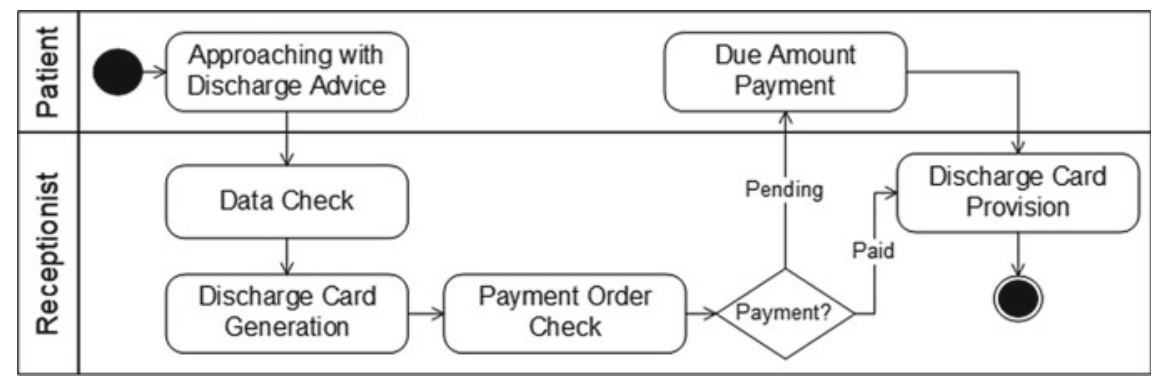

Fig. 12.8 UML activity model of hospital information management process example. Discharging

\subsubsection{UML Sequence Models of Hospital Information Management Process Example}

UML Sequence model is an interaction model that defines how operations are implemented. This model fixates the interaction between objects in the context of a collaboration. UML Sequence model is time focus and it shows the order of the interaction visually by using the vertical axis of the diagram to deliver time what messages are sent and when $[3,7,8]$.

According previously described UML Use Case and UML Activity models there is possible to identify at least three different UML Sequence models: Patient Admission, Tests and Treatment, and Discharging.

\section{UML Sequence Model: Patient Admission}

First UML Sequence Model generated from EM is Patient Admission, where four participants-Lifelines take part: Patient, Receptionist, Database and Ward.

Table 12.8 presents UML Sequence model elements generated from Enterprise model of Hospital Information Management process example, Patient Admission part. In Enterprise Model all information related with actors and their collaboration is stored. There are four actors - process participants, which are called Lifelines in UML Sequence model: persons_-Patient, Receptionist, subject—Database, objectWard. Patient registers to the hospital, Receptionist enters gathered data, Patient requests for the ward, Receptionist checks availability and confirms or denies ward availability.

Figure 12.9 presents UML Sequence model of Hospital Information Management process example, Patient Admission part generated step by step from Enterprise model through UML Sequence model transformation algorithm [9].

\section{UML Sequence Model: Tests and Treatment}

Second UML Sequence Model generated from EM is Tests and Treatment, where four participants-Lifelines take part: Patient, Doctor, Operation and Test.

Table 12.9 presents UML Sequence model elements generated from Enterprise model of Hospital Information Management process example, Test and Treatment 
Table 12.8 UML sequence model elements generated from enterprise model of hospital information management process example, patient admission $[3,7,8]$

\begin{tabular}{|c|c|c|c|}
\hline $\begin{array}{l}\text { Enterprise model } \\
\text { element }\end{array}$ & $\begin{array}{l}\text { UML sequence model } \\
\text { element }\end{array}$ & $\begin{array}{l}\text { Hospital information } \\
\text { management process } \\
\text { example }\end{array}$ & Description \\
\hline \multirow[t]{4}{*}{ Actor } & \multirow[t]{4}{*}{ Lifeline } & Patient & \multirow{4}{*}{$\begin{array}{l}\text { There are four actors, in } \\
\text { UML Sequence model } \\
\text { four Lifelines, which are } \\
\text { shown using a symbol } \\
\text { that consists of a } \\
\text { rectangle forming its } \\
\text { "head" followed by a } \\
\text { vertical line and these } \\
\text { lines represent the } \\
\text { lifetime of the } \\
\text { actor-participant of the } \\
\text { process }\end{array}$} \\
\hline & & Receptionist & \\
\hline & & Database & \\
\hline & & Ward & \\
\hline \multirow[t]{11}{*}{ Process, function } & \multirow[t]{11}{*}{ Message } & Register(data) & \multirow{11}{*}{$\begin{array}{l}\text { There are eleven } \\
\text { messages, related with } \\
\text { actors and they define a } \\
\text { communication between } \\
\text { these actors }\end{array}$} \\
\hline & & Addnew(data) & \\
\hline & & Return & \\
\hline & & Return & \\
\hline & & Wardrequest() & \\
\hline & & Availabilitycheck() & \\
\hline & & Return(status) & \\
\hline & & $\begin{array}{l}\text { [not available] } \\
\text { return(n/a) }\end{array}$ & \\
\hline & & $\begin{array}{l}\text { [if available] } \\
\text { wardupdate(data) }\end{array}$ & \\
\hline & & Return & \\
\hline & & Return(noward) & \\
\hline Business rules & Execution specification & $\begin{array}{l}\text { Five execution } \\
\text { specifications }\end{array}$ & $\begin{array}{l}\text { Each of five executions } \\
\text { specification element } \\
\text { represents a period in } \\
\text { the actor's lifetime }\end{array}$ \\
\hline
\end{tabular}

part. In EM all information related with actors-lifelines and their collaboration is stored. There are four actors-process participants, which are called Lifelines in UML Sequence model: persons-Patient, Receptionist, objects-Operation, Test. Doctor performs check-up and prescribes medicine, if necessary prescribes test, Patient provides samples and gets reports, Doctor reviews reports and prescribes more medicine or prescribes operation if necessary, prescribes more tests and operates.

Figure 12.10 presents UML Sequence model of Hospital Information Management process example, Test and Treatment part generated step by step from Enterprise model through UML Sequence model transformation algorithm [9]. 


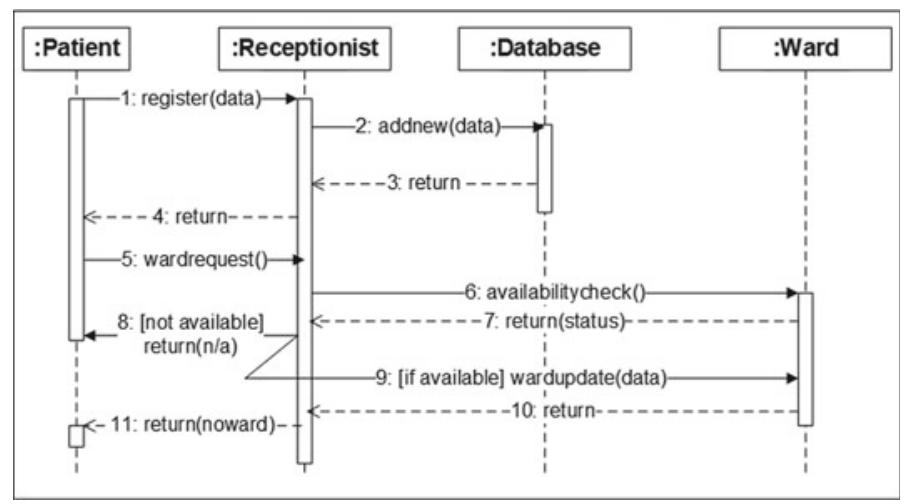

Fig. 12.9 UML sequence model of hospital information management process example, patient admission

\section{UML Sequence Model: Discharging}

Third UML Sequence Model generated from EM is Discharging, where five participants_Lifelines take part: Doctor, Patient, Reception, Database and Ward.

Table 12.10 presents UML Sequence model elements generated from Enterprise model of Hospital Information Management process example, Discharging part. In EM all information related with lifelines and their cooperation is stored. There are five actors_-process participants, which are called Lifelines in UML Sequence model: persons-Patient, Doctor, subjects-Reception, Database, object-Ward. Doctor provides discharge advice, Patient requests for discharge, Reception checks information related with payments, inquires for payment, Patient makes the payment, Reception updates financial information in Database, provides Receipt, Reception updates discharge information and information related with ward, in the end Reception provides Discharge card.

Figure 12.11 presents UML Sequence model of Hospital Information Management process example, Discharging part generated step by step from Enterprise model through UML Sequence model transformation algorithm [9].

\subsubsection{UML State Models of Hospital Information Management Process Example}

UML State Model demonstrates the diverse states of an entity. State model can also show how an entity responds to various events by changing from one state to another $[3,7,8]$.

According previously described UML models there is possible to identify at least three different UML State models describing states of: Patient, Doctor and Ward. 
Table 12.9 UML sequence model elements generated from enterprise model of hospital information management process example, test and treatment $[3,7,8]$

\begin{tabular}{|c|c|c|c|}
\hline $\begin{array}{l}\text { Enterprise model } \\
\text { element }\end{array}$ & $\begin{array}{l}\text { UML sequence } \\
\text { model element }\end{array}$ & $\begin{array}{l}\text { Hospital information } \\
\text { management process } \\
\text { example }\end{array}$ & Description \\
\hline \multirow[t]{4}{*}{ Actor } & \multirow[t]{4}{*}{ Lifeline } & Patient & \multirow{4}{*}{$\begin{array}{l}\text { There are four actors, in } \\
\text { UML Sequence model four } \\
\text { Lifelines, which are shown } \\
\text { using a symbol that consists } \\
\text { of a rectangle forming its } \\
\text { "head" followed by a } \\
\text { vertical line and these lines } \\
\text { represent the lifetime of the } \\
\text { actor-participant of the } \\
\text { process }\end{array}$} \\
\hline & & Doctor & \\
\hline & & Operation & \\
\hline & & test & \\
\hline \multirow[t]{13}{*}{ Process, function } & \multirow[t]{13}{*}{ Message } & Performcheckup() & \multirow{13}{*}{$\begin{array}{l}\text { There are thirteen } \\
\text { messages, related with } \\
\text { actors and they define a } \\
\text { communication between } \\
\text { these actors }\end{array}$} \\
\hline & & Return & \\
\hline & & Prescribemedicine() & \\
\hline & & Return & \\
\hline & & Prescribetest() & \\
\hline & & Providesamples(samples) & \\
\hline & & Return(report) & \\
\hline & & Inquirereview(reports) & \\
\hline & & Prescribemedicine() & \\
\hline & & Prescribeoperation() & \\
\hline & & Moretest() & \\
\hline & & Getoperated() & \\
\hline & & Operate() & \\
\hline Business rules & $\begin{array}{l}\text { Execution } \\
\text { specification }\end{array}$ & $\begin{array}{l}\text { Five execution } \\
\text { specifications }\end{array}$ & $\begin{array}{l}\text { Each of five executions } \\
\text { specification element } \\
\text { represents a period in the } \\
\text { actor's lifetime }\end{array}$ \\
\hline
\end{tabular}

\section{UML State Model: Patient}

First UML State Model generated from EM describes states of Patient.

Table 12.11 presents UML State model elements generated from Enterprise model of Hospital Information Management process example, Patient part. In Enterprise Model all information related with processes, functions and their states is stored. This model is from Patient's perspective. In the model these elements are presented: initial state which starts the process, first state Patient registered, it's state changes after doctors visit: patient receives treatment, additional doctor's visit, after which doctor advices discharge procedure and patient's state changes again, patient is discharged, process ends with final state. 


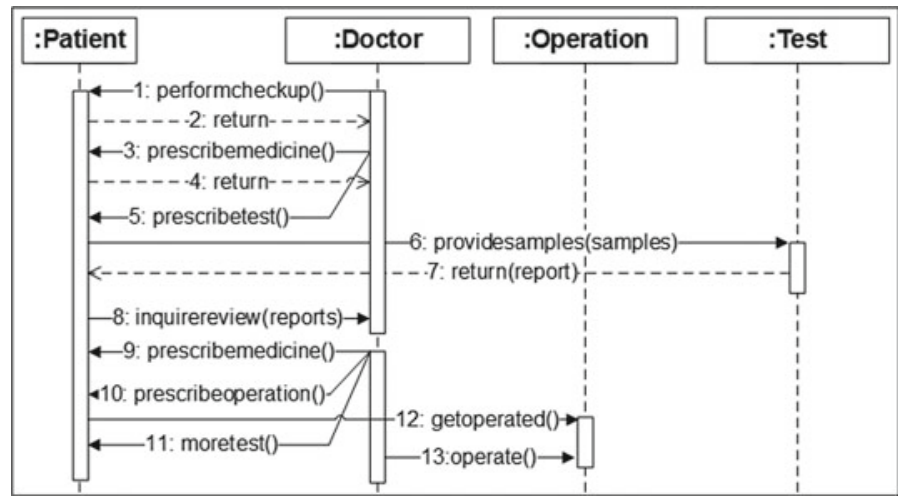

Fig. 12.10 UML sequence model of hospital information management process example, test and treatment

Figure 12.12 presents UML State model of Hospital Information Management process example, Patient part.

\section{UML State Model: Doctor}

Second UML State Model generated from EM describes states of Doctor.

Table 12.12 presents UML State model elements generated from Enterprise model of Hospital Information Management process example, Doctor part. In Enterprise Model all information related with processes, functions and their states is stored. This model is from Doctor's perspective. In the model these elements are presented: initial state which starts the process, first state Doctor registered, it's state changes after patient registers to the visit: Doctor prescribes treatment, check's up treatment results, after patient's discharge procedure Doctor's state changes, he is not needed for this particular patient, process ends with final state.

Figure 12.13 presents UML State model of Hospital Information Management process example, Doctor part.

\section{UML State Model: Ward}

Third UML State Model generated from EM describes states of Ward.

Table 12.13 presents UML State model elements generated from Enterprise model of Hospital Information Management process example, Ward part. In Enterprise Model all information related with processes, functions and their state is stored. This model is from Ward's perspective. In the model these elements are presented: initial state which starts the process, first state means ward is free, its state changes after request to occupy; after patient is discharged ward state changes again to free.

Figure 12.14 presents UML State model of Hospital Information Management process example, Ward part.

With the help of Hospital Information Management process example result of four UML models: Use Case, Activity, Sequence and State generation from Enterprise Model through transformation algorithms is presented in detailed way, all models 
Table 12.10 UML sequence model elements generated from enterprise model of hospital information management process example, discharging [3, 7, 8]

\begin{tabular}{|c|c|c|c|}
\hline $\begin{array}{l}\text { Enterprise } \\
\text { model element }\end{array}$ & $\begin{array}{l}\text { UML sequence } \\
\text { model element }\end{array}$ & $\begin{array}{l}\text { Hospital information } \\
\text { management process example }\end{array}$ & Description \\
\hline \multirow[t]{5}{*}{ Actor } & \multirow[t]{5}{*}{ Lifeline } & Doctor & \multirow{5}{*}{$\begin{array}{l}\text { There are five actors, in } \\
\text { UML Sequence model } \\
\text { four Lifelines, which are } \\
\text { shown using a symbol } \\
\text { that consists of a } \\
\text { rectangle forming its } \\
\text { "head" followed by a } \\
\text { vertical line and these } \\
\text { lines represent the } \\
\text { lifetime of the } \\
\text { actor-participant of the } \\
\text { process }\end{array}$} \\
\hline & & Patient & \\
\hline & & Reception & \\
\hline & & Database & \\
\hline & & Ward & \\
\hline \multirow{15}{*}{$\begin{array}{l}\text { Process, } \\
\text { function }\end{array}$} & \multirow{15}{*}{ Message } & Dischargeadvice() & \multirow{15}{*}{$\begin{array}{l}\text { There are fifteen } \\
\text { messages, related with } \\
\text { actors and they define a } \\
\text { communication between } \\
\text { these actors }\end{array}$} \\
\hline & & Requestdiscgarge() & \\
\hline & & Checkdues(patientid) & \\
\hline & & Return(dues) & \\
\hline & & Askpayment(amount) & \\
\hline & & Paydues(amount,patientid) & \\
\hline & & Update(amount,patientid) & \\
\hline & & Return(receipt) & \\
\hline & & Return(receipt) & \\
\hline & & Return & \\
\hline & & Updatedischargedata(patientid) & \\
\hline & & Updateward() & \\
\hline & & Return & \\
\hline & & Return & \\
\hline & & Grantdicharge(dichargecard) & \\
\hline \multirow[t]{2}{*}{ Business rules } & $\begin{array}{l}\text { Execution } \\
\text { specification }\end{array}$ & Ten Execution Specifications & \multirow{2}{*}{$\begin{array}{l}\text { Each of ten executions } \\
\text { specification element } \\
\text { represents a period in the } \\
\text { actor's lifetime. Each } \\
\text { parallels defines } \\
\text { potentially parallel } \\
\text { execution of behaviors } \\
\text { of the operands of the } \\
\text { combined fragment }\end{array}$} \\
\hline & Parallel & Two parallels & \\
\hline
\end{tabular}




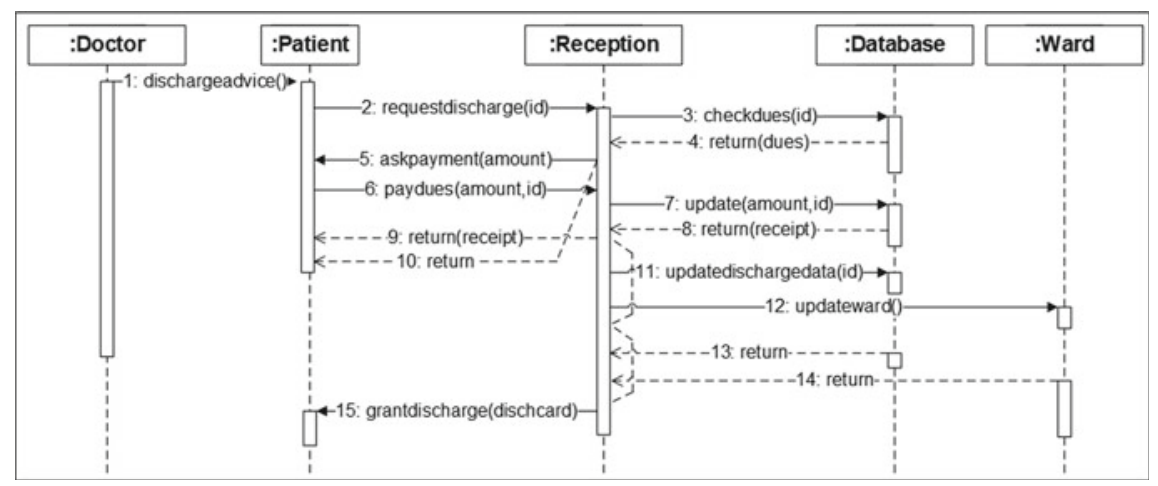

Fig. 12.11 UML sequence model of hospital information management process example, discharging

Table 12.11 UML state model elements generated from enterprise model of hospital information management process example, patient $[3,7,8]$

\begin{tabular}{|c|c|c|c|}
\hline $\begin{array}{l}\text { Enterprise model } \\
\text { element }\end{array}$ & $\begin{array}{l}\text { UML state model } \\
\text { element }\end{array}$ & $\begin{array}{l}\text { Hospital information } \\
\text { management process } \\
\text { example }\end{array}$ & Description \\
\hline \multirow[t]{3}{*}{ Process, function } & \multirow[t]{3}{*}{ Transition } & Doctor visit & \multirow{3}{*}{$\begin{array}{l}\text { Transitions from one } \\
\text { state to the next respond } \\
\text { to the activities, events, } \\
\text { what causes the state's } \\
\text { change }\end{array}$} \\
\hline & & Doctor review & \\
\hline & & Issue to discharge & \\
\hline \multirow[t]{3}{*}{ Information flow } & \multirow[t]{3}{*}{ Simple state } & Patient registered & \multirow{3}{*}{$\begin{array}{l}\text { Internal activities } \\
\text { compartment holds a list } \\
\text { of internal actions or } \\
\text { state (do) activities } \\
\text { (behaviours) that are } \\
\text { performed while the } \\
\text { element is in the state }\end{array}$} \\
\hline & & Treatment in progress & \\
\hline & & Discharged & \\
\hline \multirow[t]{2}{*}{ Business rules } & \multirow{2}{*}{$\begin{array}{l}\text { Initial and final } \\
\text { states }\end{array}$} & Initial state & \multirow{2}{*}{$\begin{array}{l}\text { Initial and final states are } \\
\text { a special kind of states } \\
\text { signifying the beginning } \\
\text { and closing processes of } \\
\text { defined states }\end{array}$} \\
\hline & & Final state & \\
\hline
\end{tabular}

define same example, but from different perspectives. In almost each subsection of the described example there are more than one model of the same type presented: generated UML Use Case model presents all participants (Actors), which are involved in Hospital Information Management process and their functions/processes (Use Cases); generated UML Activity models illustrate different activities from different perspectives (Registration, Ward Assignation, Medical Tests, Treatment process and Discharging) of the same example, and it is not final list of possible models of 
Fig. 12.12 UML state model of hospital information management process example, patient

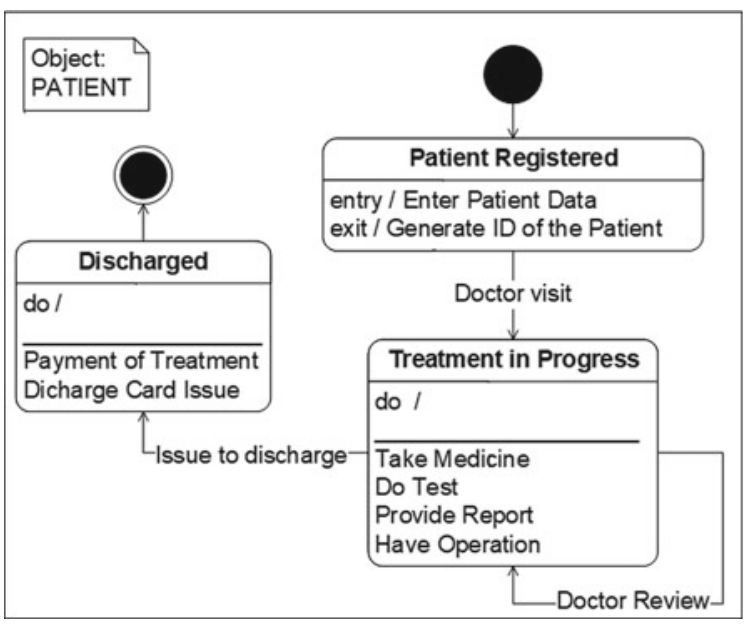

Table 12.12 UML state model elements generated from enterprise model of hospital information management process example, doctor $[3,7,8]$

\begin{tabular}{|c|c|c|c|}
\hline $\begin{array}{l}\text { Enterprise model } \\
\text { element }\end{array}$ & $\begin{array}{l}\text { UML state model } \\
\text { element }\end{array}$ & $\begin{array}{l}\text { Hospital information } \\
\text { management process } \\
\text { example }\end{array}$ & Description \\
\hline \multirow[t]{3}{*}{ Process, function } & \multirow[t]{3}{*}{ Transition } & Patient Registered & \multirow{3}{*}{$\begin{array}{l}\text { Transitions from one } \\
\text { state to the next respond } \\
\text { to the activities, events, } \\
\text { what causes the state's } \\
\text { change }\end{array}$} \\
\hline & & Patient (re)Checkup & \\
\hline & & $\begin{array}{l}\text { Planned leave of the } \\
\text { Doctor }\end{array}$ & \\
\hline \multirow[t]{3}{*}{ Information flow } & \multirow[t]{3}{*}{ Simple state } & Doctor registered & \multirow{3}{*}{$\begin{array}{l}\text { Internal activities } \\
\text { compartment holds a list } \\
\text { of internal actions or } \\
\text { state (do) activities } \\
\text { (behaviours) that are } \\
\text { performed while the } \\
\text { element is in the state }\end{array}$} \\
\hline & & Appointing treatment & \\
\hline & & Doctor inactive & \\
\hline \multirow[t]{2}{*}{ Business rules } & \multirow{2}{*}{$\begin{array}{l}\text { Initial and final } \\
\text { states }\end{array}$} & Initial state & \multirow{2}{*}{$\begin{array}{l}\text { Initial and final states are } \\
\text { a special kind of states } \\
\text { signifying the beginning } \\
\text { and closing processes of } \\
\text { defined states }\end{array}$} \\
\hline & & Final state & \\
\hline
\end{tabular}

the same type; generated UML Sequence model also define sequence processes and functions sequences from different perspectives (Patient Admission, Tests and Treatment, Discharging) of the same example, and it is also not final list of possible UML Sequence models; generated UML State model describe different states from the perspectives of objects (Patient, Doctor and Ward), and states of more objects of the same example can be generated. 
Fig. 12.13 UML state model of hospital information management process example, patient

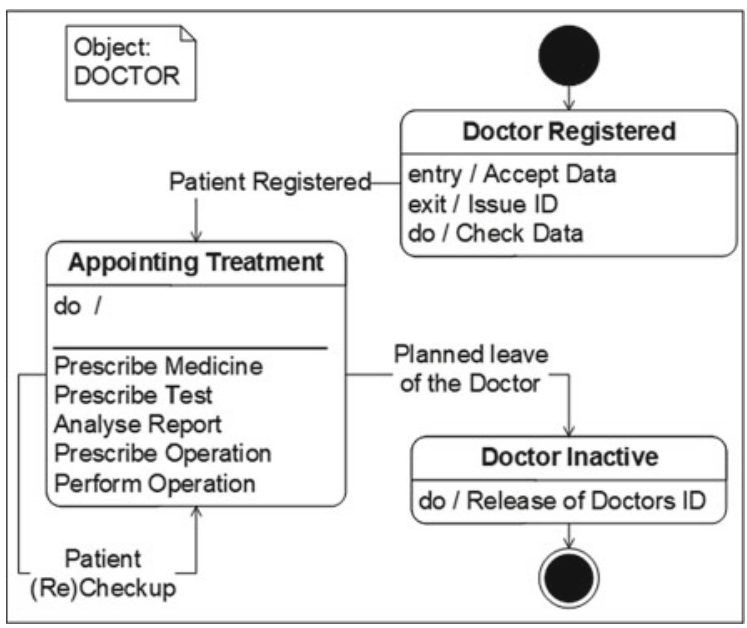

Table 12.13 UML state model elements generated from enterprise model of hospital information management process example, Ward [3, 7, 8]

\begin{tabular}{|c|c|c|c|}
\hline $\begin{array}{l}\text { Enterprise model } \\
\text { element }\end{array}$ & $\begin{array}{l}\text { UML state model } \\
\text { element }\end{array}$ & $\begin{array}{l}\text { Hospital information } \\
\text { management process } \\
\text { example }\end{array}$ & Description \\
\hline \multirow[t]{2}{*}{ Process, function } & \multirow[t]{2}{*}{ Transition } & Request to occupy & \multirow{2}{*}{$\begin{array}{l}\text { Transitions from one } \\
\text { state to the next respond } \\
\text { to the activities, events, } \\
\text { what causes the state's } \\
\text { change }\end{array}$} \\
\hline & & Patient discharged & \\
\hline \multirow[t]{2}{*}{ Information flow } & \multirow[t]{2}{*}{ Simple state } & Free & \multirow[b]{2}{*}{$\begin{array}{l}\text { Internal activities } \\
\text { compartment holds a list } \\
\text { of internal actions or } \\
\text { state (do) activities } \\
\text { (behaviours) that are } \\
\text { performed while the } \\
\text { element is in the state }\end{array}$} \\
\hline & & Occupied & \\
\hline Business rules & $\begin{array}{l}\text { Initial and final } \\
\text { states }\end{array}$ & Initial state & $\begin{array}{l}\text { Initial state is a special } \\
\text { kind of states signifying } \\
\text { the beginning process of } \\
\text { defined states }\end{array}$ \\
\hline
\end{tabular}

Provided example of Hospital Information Management process shows and confirms, that it is not the final amount of UML models, which can be generated from EM, there are more different perspectives for UML models generation of the same example. As stated previously, knowledge-based Enterprise model which stores verified and validated data of a specific problem domain is enough data storage for generation various UML models. 
Fig. 12.14 UML state model of hospital information management process example, ward

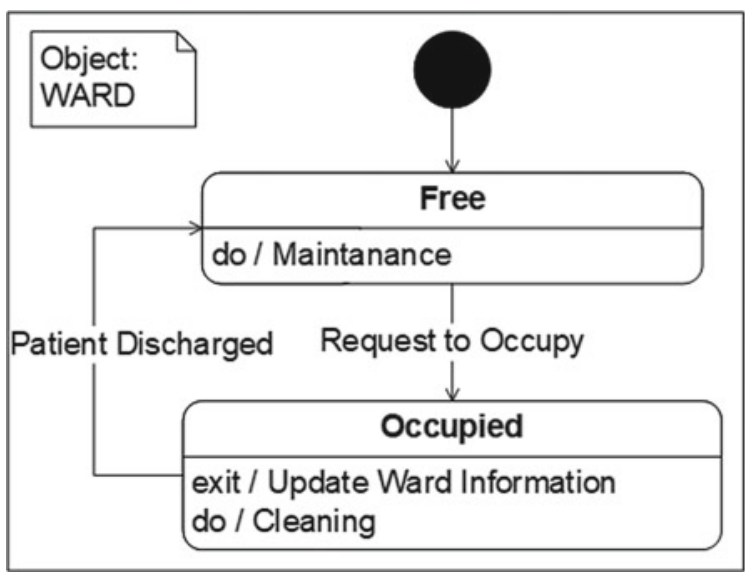

\subsection{Conclusions}

The first part of the paper presents the structure of knowledge-based Enterprise model, by defining all its components and their relations.

The second part deals with the presentation of UML models generation form Enterprise model top level transformation algorithm, which is defined structurally step by step. In this section also part of Enterprise model elements, necessary for particular example of the research, are presented and their descriptions are also provided.

The third part presents Hospital Information Management process example, which data is stored in knowledge-based Enterprise model and used for UML models generation. There are defined four types of UML dynamic models in details, which represent data of chosen example in different perspectives. Each presented UML model is generated through certain transformation algorithms introduced in previous researches.

Each subsection describes different type of UML dynamic model and presents particular type of UML models variations: same UML model type, but different data used for different perspectives. All these UML models are generated based the knowledge stored in Enterprise model.

The defined Hospital Information Management process example shows that verified and validated knowledge stored in Enterprise model is sufficient for UML models generation process; that stored in Enterprise model elements are enough to transfer all UML models elements, despite the perspective of certain UML model. Using UML models generated from Enterprise Model full IS development life cycle design stage can be implemented as knowledge-based process.

Acknowledgements This publication is based upon work from COST Action "European Network for cost containment and improved quality of health care-CostCares" (CA15222), supported by COST (European Cooperation in Science and Technology) 
COST (European Cooperation in Science and Technology) is a funding agency for research and innovation networks. Our Actions help connect research initiatives across Europe and enable scientists to grow their ideas by sharing them with their peers. This boosts their research, career and innovation.

https://www.cost.eu

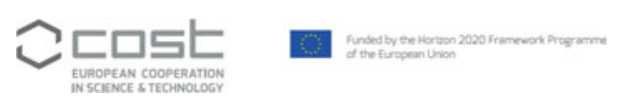

\section{References}

1. Dunkel, J., Bruns, R.: Model-driven architecture for mobile applications. In: Proceedings of the 10th Inter-national Conference on Business Information Systems (BIS), Vol. 4439/2007, pp. 464-477 (2007)

2. Eichelberger, H., Eldogan, Y., Schmid, K.A.: Comprehensive Analysis of UML Tools, their Capabilities and Compliance. Software Systems Engineering. Universität Hildesheim. versio 2.0 (2011)

3. Jacobson, I., Rumbaugh, J., Booch, G.: Unified Modeling Language User Guide, 2nd edn. Addison-Wesley Professional. ISBN: 0321267974 (2005)

4. Jenney, J.: Modern methods of systems engineering: with an introduction to pattern and model based methods. ISBN-13:978-1463777357 (2010)

5. Sajja, P.S., Akerkar, R.: Knowledge-Based Systems for Development. Advanced Knowledge Based Systems: Model, Applications \& Research, Vol. 1 (2010)

6. Gudas, S.: Architecture of Knowledge-Based Enterprise Management Systems: a Control View. In: Proceedings of the 13th world multiconference on systemics, cybernetics and informatics (WMSCI2009), ) July 10-13, Orlando, Florida, USA, Vol. III, pp. 161-266. ISBN -10: 19934272-61-2 (Volume III). ISBN -13: 978-1-9934272-61-9 (2009)

7. OMG UML (2019) Unified Modeling Language version 2.5.1. Unified Modelling. https://www. omg.org/spec/UML/About-UML/

8. UML Diagrams: UML diagrams characteristic (2012). http://www.uml-diagrams.org

9. Veitaitė, I., Lopata, A.: Transformation algorithms of knowledge based UML dynamic models generation. Business information systems workshops BIS 2017, Poznan, Poland, 28-30 June. Abramowicz, W, (ed.), Lecture Notes in Business Information Processing, Vol. 303. Springer International Publishing, Cham (2017)

10. Gudas, S.: Informacijos sistemụ inžinerijos teorijos pagrindai/Fundamentals of Information Systems Engineering Theory. (Lithuanian)Vilnius University. ISBN 978-609-459-075-7 (2012)

11. Veitaitè, I., Lopata, A.: Problem domain knowledge driven generation of UML models. In: Damaševičius, R., Vasiljevienè, G. (eds.), Information And Software Technologies: 24th International Conference, ICIST 2018, Vilnius, Lithuania, October 4-6, 2018: Proceedings. Springer, Cham (2018)

12. Veitaite, I., Lopata, A.: Knowledge-Based Transformation Algorithms of UML Dynamic Models Generation from Enterprise Model. In: Dzemyda, G., Bernatavičienè, J., Kacprzyk, J. (eds.), Data Science: New Issues, Challenges and Applications. Studies in Computational Intelligence, Vol. 869. Springer, Cham (2020)

13. Veitaite, I., Lopata, A.: Knowledge-Based Generation of the UML Dynamic Models from the Enterprise Model Illustrated by the Ticket Buying Process Example. In: Lopata, A., Butkienè, R., Gudonienè, D., Sukackè, V. (eds.), Information and Software Technologies. ICIST 2020. Communications in Computer and Information Science, Vol. 1283. Springer, Cham (2020). https://doi.org/10.1007/978-3-030-59506-7_3 
Open Access This chapter is licensed under the terms of the Creative Commons Attribution 4.0 International License (http://creativecommons.org/licenses/by/4.0/), which permits use, sharing, adaptation, distribution and reproduction in any medium or format, as long as you give appropriate credit to the original author(s) and the source, provide a link to the Creative Commons license and indicate if changes were made.

The images or other third party material in this chapter are included in the chapter's Creative Commons license, unless indicated otherwise in a credit line to the material. If material is not included in the chapter's Creative Commons license and your intended use is not permitted by statutory regulation or exceeds the permitted use, you will need to obtain permission directly from the copyright holder.

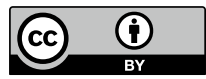

